Sai G. Yarram • Michael A. Dipietro

Kathleen Graziano • George B. Mychaliska

Peter J. Strouse

\title{
Bilateral giant abdominoscrotal hydroceles complicated by appendicitis
}

Received: 28 June 2005 / Accepted: 21 July 2005/Published online: 14 September 2005

(C) Springer-Verlag 2005

\begin{abstract}
Abdominoscrotal hydrocele is a rare entity, with fewer than 100 cases reported in children. Bilateral abdominoscrotal hydroceles are even less common, with 14 cases reported in children. Various complications of abdominoscrotal hydrocele have been reported in the literature. We present a 4-month-old boy with bilateral giant abdominoscrotal hydroceles who developed appendicitis apparently because of obstruction from the right hydrocele. We discuss the various imaging modalities used to establish the diagnosis and plan the operative approach.
\end{abstract}

Keywords Bilateral $\cdot$ Abdominoscrotal hydrocele $\cdot \mathrm{CT}$

\section{Introduction}

Abdominoscrotal hydrocele is a rare form of hydrocele originally described by Dupuytren in 1834 [1]. It was first described in children in 1861 [2]. As uncommon as this condition is, with fewer than 100 cases reported in children, bilateral abdominoscrotal hydroceles are even rarer. There have been fewer than 10 reports, with 14 cases of bilateral abdominoscrotal hydroceles reported in the literature since the initial report by Squire and Gough in 1988 [3]. Several papers have been written about the pathogenesis, complications and surgical approach to repair this condition [4-8]. Appendicitis as an associated complication is a new entity.

S. G. Yarram $(\bowtie) \cdot$ M. A. Dipietro · P. J. Strouse Department of Radiology, University of Michigan, 1500 E. Medical Center Drive, 48109 Ann Arbor, MI, USA

E-mail: syarram@med.umich.edu

Tel.: + 1-734-6154924

Fax: + 1-734-6479339

K. Graziano · G. B. Mychaliska

Department of Surgery, University of Michigan, Ann Arbor, MI 48109, USA

\section{Case report}

A 4-month-old boy was referred with a history of an asymptomatic palpable cystic abdominal mass. Abdominal sonogram at another institution demonstrated a cystic intra-abdominal mass. His birth history was pertinent for being a full-term infant who was delivered by cesarean section because of position and for the presence of bilateral scrotal hydroceles since birth (Fig. 1). Abdominal US performed at our institution demonstrated a bilobed cystic structure measuring $5.5 \times 3.7 \times 5.5 \mathrm{~cm}(\mathrm{CC}, \mathrm{AP}$, and TR) and $8.6 \times 5.1 \times 5.7 \mathrm{~cm}$ (CC, AP, and TR) (Fig. 2). It was not appreciated that this intra-abdominal mass communicated with the scrotal hydroceles. The differential diagnosis included a mesenteric cyst, intestinal duplication, and lymphangioma. To better characterize the lesion, a multidetector CT was performed; that better delineated the cystic abdominal mass and established the diagnosis (Fig. 3). Coronal and sagittal reformatted images of the abdomen and pelvis demonstrated bilateral dumbbell-shaped fluid attenuation structures, each communicating with its ipsilateral hemiscrotum spanning a length of $13 \mathrm{~cm}$ (right) and $15 \mathrm{~cm}$ (left) (Fig. 4).

After the diagnostic CT, the patient was scheduled for elective operative repair of the bilateral abdominoscrotal hydroceles. However, he presented the day before the scheduled surgery with irritability, vomiting, feeding intolerance, and low-grade fever. On physical examination, he was noted to be irritable and uncomfortable, with a temperature of $37.4^{\circ} \mathrm{C}$. His abdomen was mildly tender, and the cystic masses were palpable. There were no peritoneal signs. The remainder of the physical examination was unremarkable. Plain radiographs performed at that time were unremarkable. White blood cell count was elevated, at $27,800 \mathrm{~mm}^{-3}$. Urinalysis was unremarkable.

Based on the preoperative imaging, we planned a combined laparoscopic and inguinal approach. A 5-mm laparoscope was inserted through the umbilicus. We 


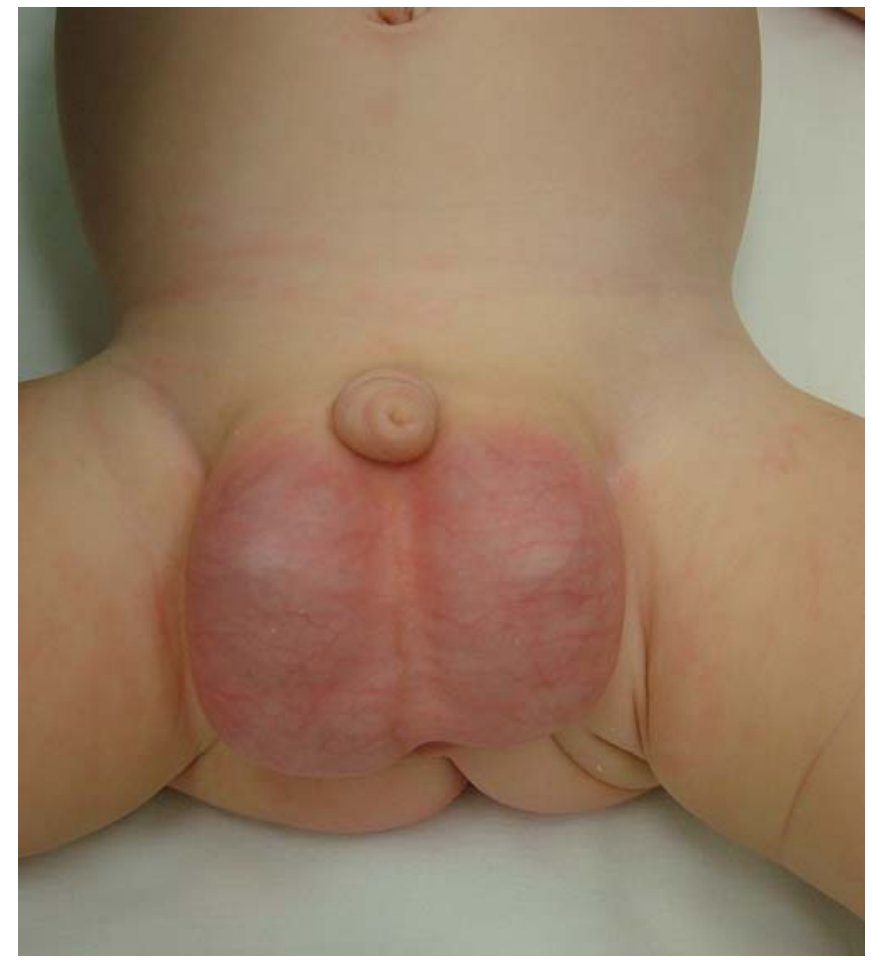

Fig. 1 Preoperative photograph of the patient shows marked enlargement and mild erythema of the scrotum

unexpectedly found purulent fluid in the right paracolic gutter with dense adhesions between the right abdominoscrotal hydrocele and the cecum. The appendix was not visible. Given the findings, the procedure was converted to an open surgery that revealed an inflamed and fibrotic right intra-abdominal hydrocele sac compressing a retrocecal non-perforated appendix. Partial drainage of the hydroceles bilaterally facilitated dissection. The vas deferens and testicular vessels were splayed and densely adhered to the abdominal hydrocele sacs and required careful dissection to prevent injury. The abdominal component of each hydrocele was then inverted through the bilateral inguinal incisions, and the hydrocele sacs were removed completely. An appendectomy was performed along with the repair of the abdominoscrotal hydroceles (Fig. 5). The patient's postoperative course was unremarkable. Histologic sections of the specimen revealed organizing peritonitis in the appendix and acutely inflamed fibroconnective tissue in the excised right abdominoscrotal hydrocele. No appendicolith was present.

\section{Discussion}

As the name implies, an abdominoscrotal hydrocele communicates between the abdomen and the scrotum. It is a rare congenital inguinal abnormality for which three theories of pathogenesis exist. According to Dupuytren, excessive distention of the tunica vaginalis displaces the hydrocele superiorly through the inguinal canal into the abdomen to form the abdominal component [1]. A second theory proposes that the scrotal hydrocele extends into the abdomen through a valve-like action of the processus vaginalis when intrascrotal pressure becomes high. The third theory postulates the presence of a preformed congenital peritoneal diverticulum with an underlying anatomic abnormality of the preexisting abdominal sac $[4,8,9]$.

Regardless of the pathogenesis of abdominoscrotal hydrocele, some important complications have been reported. These include hydroureteronephrosis, paratesticular malignant mesothelioma and lower extremity edema [5-7]. To this list of complications we add appendicitis as occurred in this child. Appendicitis is relatively rare in children 4 months of age. Although it is possible that this child developed appendicitis de novo, at surgery the inflamed non-perforated retrocecal appendix was adherent and compressed by the right abdominoscrotal hydrocele. No appendicolith was found at surgery or on pathologic examination. We postulate that extrinsic compression of the appendiceal lumen by the abdominoscrotal hydrocele led to the development of appendicitis.

Traditionally, abdominoscrotal hydrocele has been evaluated and diagnosed with US [4, 9]. However, in large bilateral lesions it can be difficult to clearly delineate the anatomy and the full extent of the abnormality. Sonography with extended field-of-view capability
Fig. 2 US examination. a Axial image shows a bilobed mass with right $(R)$ and left $(L)$ components. The septation between left and right is not well seen, as it is parallel to the longitudinal axis of the US beam. b Sagittal image of the larger, leftward component of the mass $(L)$. The mass tapers inferiorly
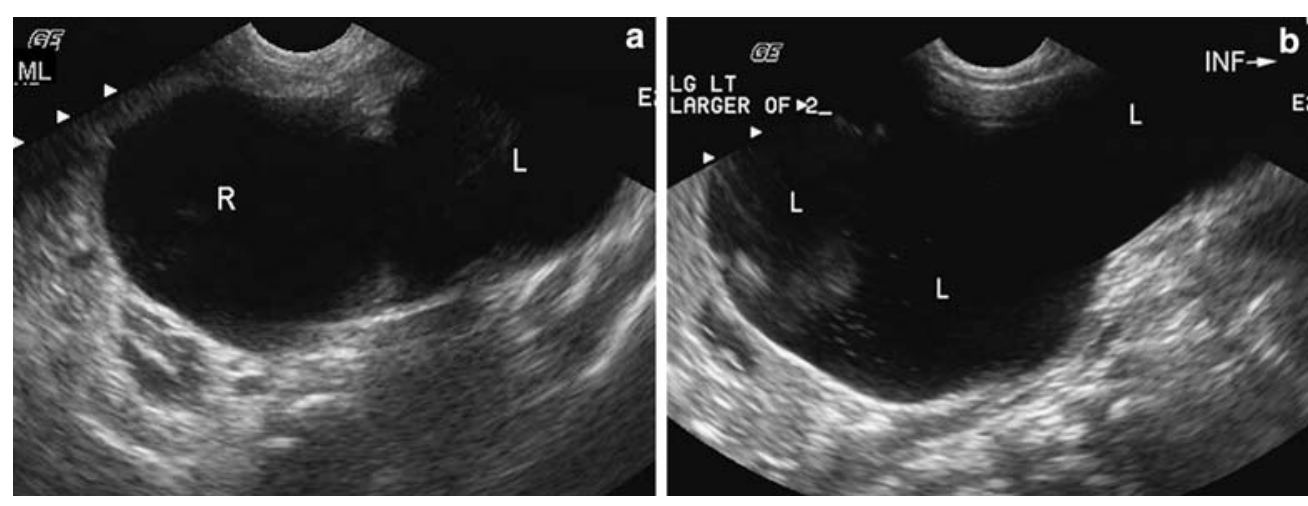


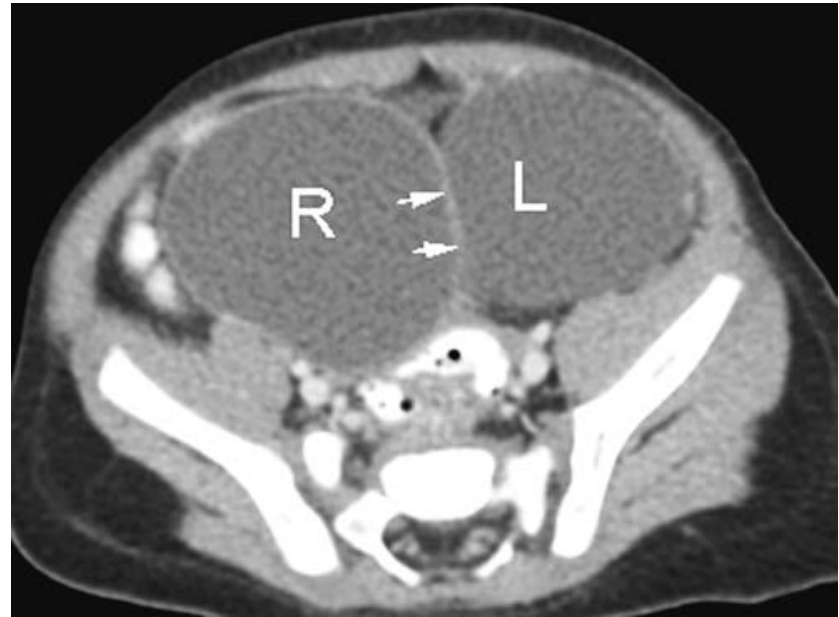

Fig. 3 Axial contrast-enhanced CT image clearly demonstrates separate right $(R)$ and left $(L)$ components of the mass. The masses abut (arrows)

would have been helpful. In addition to US, the use of $\mathrm{CT}$ and MRI to diagnose abdominoscrotal hydrocele has been reported in the literature [7, 10,11]. Depending on availability, either modality will provide the multiplanar imaging that can lead to the correct diagnosis.

If a relationship between the cystic abdominal mass and the hydrocele is not clearly defined by sonography, the traditional modality for imaging the abdomen and the scrotum in a young child, CT or MRI, should be considered. We used multidetector CT in our case because it was more readily available than MRI at the time. The coronal and sagittal reformatted images

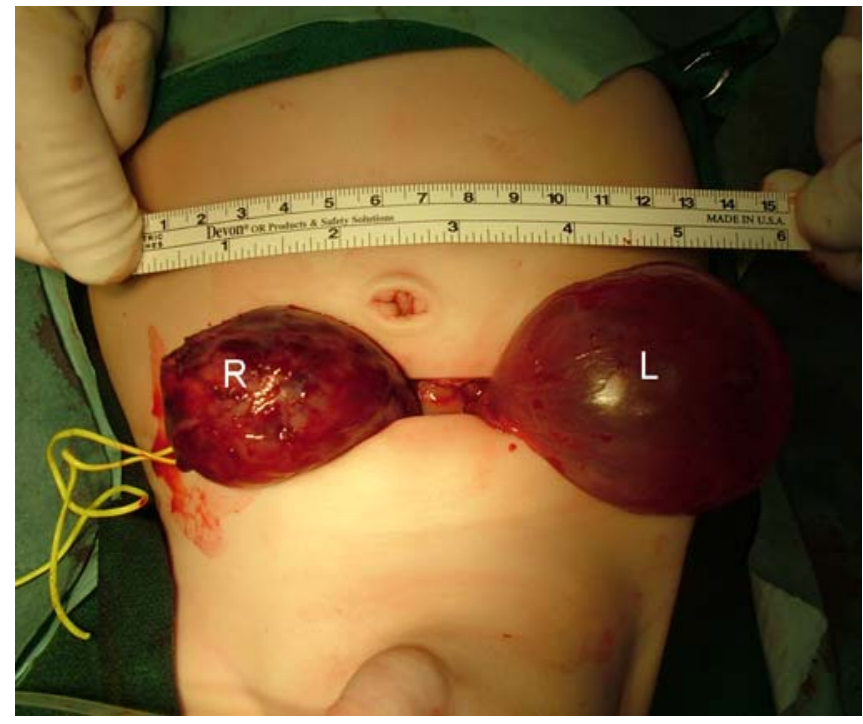

Fig. 5 Intraoperative photograph shows the right $(R)$ and left $(L)$ masses seen separately through a transverse abdominal incision

provided a broader, more panoramic view of the cystic masses and the relationship of the abdominal and scrotal components. This information might also be obtained in the sagittal plane from sonography with extended fieldof-view capability.

The operative approach to resection of an abdominoscrotal hydrocele depends on the size of the abdominal component of the sac. There are reports in the literature of resection solely through an inguinal approach [11]. After partial decompression of the hydrocele, the abdominal portion of the sac can be mobilized
Fig. 4 Reformatted CT images. a Coronal and $\mathbf{b}$ sagittal reformatted CT images show continuity of the intraabdominal portion of each mass with the corresponding hydrocele ( $R$ right, $L$ left, arrows testicles)
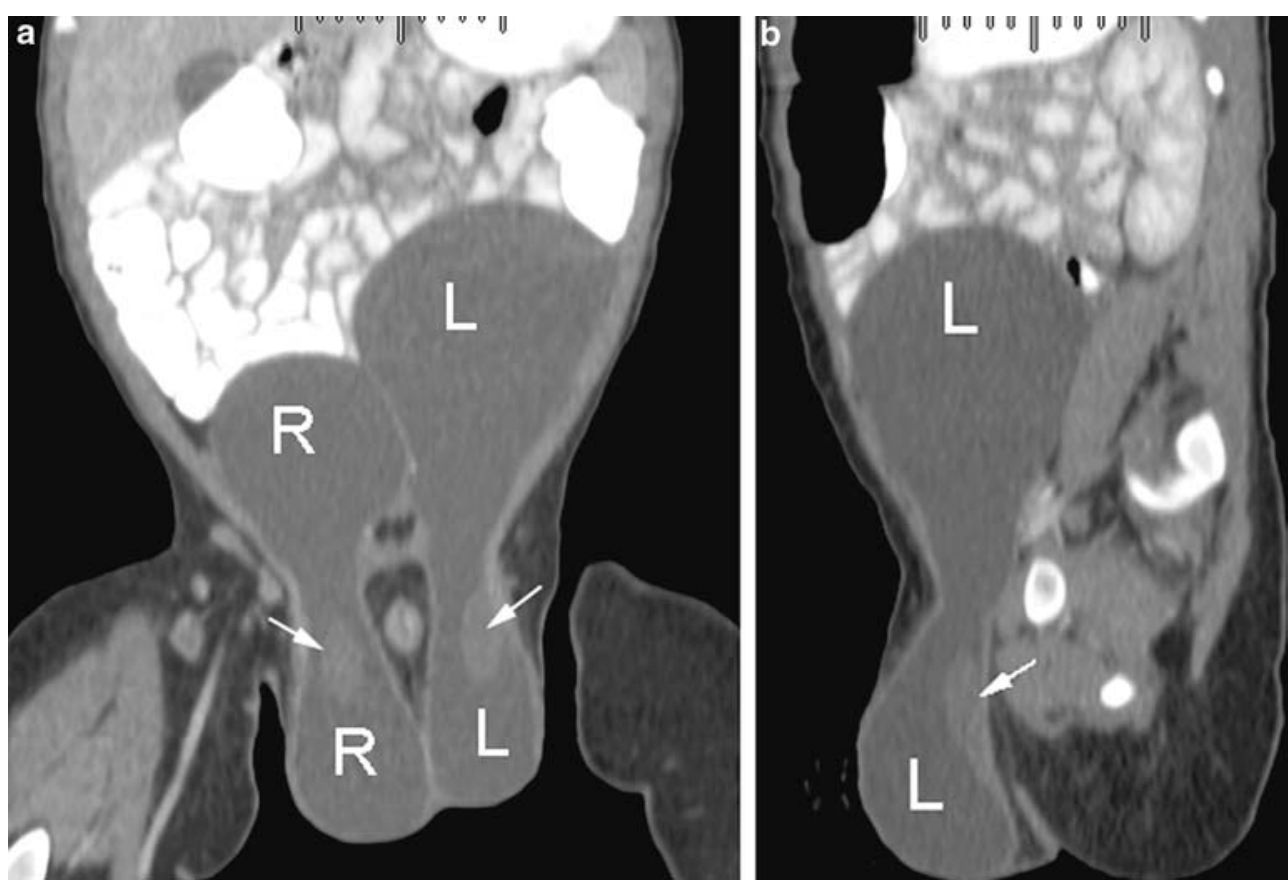
into the groin incision and resected. Other reports have suggested using a combined transabdominal and inguinal approach to accurately identify the vas deferens and testicular vessels, which might be abnormally positioned $[9,10]$. In our case, we chose to evaluate the resectability of the abdominal sacs using laparoscopy. We chose to convert to an open approach when we encountered purulent fluid and extensive inflammation in the right sac and cecum.

In conclusion, abdominoscrotal hydrocele should be considered in the differential diagnosis of a cystic lower abdominal mass in a child with a hydrocele. Diagnostic imaging modalities include sonography with extended field-of-view, and CT and MRI with imaging in sagittal or coronal planes to demonstrate a connection between the abdominal and scrotal components. An awareness of potential complications is important for efficacious treatment.

\section{References}

1. Dupuytren G (1834) Lecons orales de clinique chirugicale. Balliere, p 444
2. Syme J (1861) Abdominal hydrocele. BMJ 2:139

3. Squire R, Gough DC (1988) Abdominoscrotal hydrocele in infancy. Br J Urol 61:347-349

4. Cerrah CA, Akyuz H, Ciftlik A, et al (2001) Critical observation about the pathogenesis of abdominoscrotal hydrocele. J Pediatr Surg 36:1082-1084

5. Klin B, Efrati Y, Mor A, et al (1992) Unilateral hydroureteronephrosis caused by abdominoscrotal hydrocele. J Urol 148:384-386

6. Velasco AL, Ophoven J, Priest JR, et al (1988) Paratesticular malignant mesothelioma associated with abdominoscrotal hydrocele. J Pediatr Surg 23:1065-1067

7. Krasna IH, Solomon M, Mezrich R, et al (1992) Unilateral leg edema caused by abdominoscrotal hydrocele: elegant diagnosis by MRI. J Pediatr Surg 27:1349-1351

8. Ferro F, Spagnoli A, Lucchetti MC, et al (2000) Abdominoscrotal hydrocele: a reliable surgical technique. Urology 55:771773

9. Serels S, Kogan S (1996) Bilateral giant abdominoscrotal hydroceles in childhood. Urology 47:763-765

10. Schlup S, Hanquinet S, Merak D, et al (2002) MRI and MRA of a giant hydrocele in an infant. Pediatr Radiol 32:885-887

11. Fenton LZ, McCabe KJ (2002) Giant unilateral abdominoscrotal hydrocele. Pediatr Radiol 32:882-884 